1

دراسة بعض خواص الترب الملحية ومحتواها من الفطريات في بعض أسباخ مدينة مصراتة

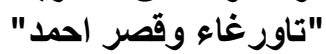

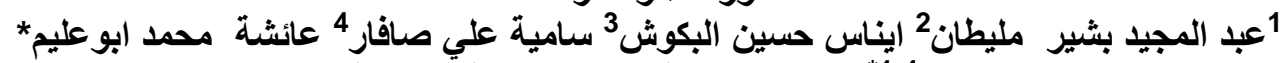

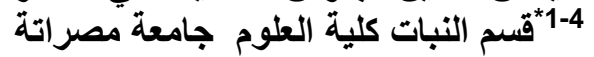
aefmsr2012@yahoo.com

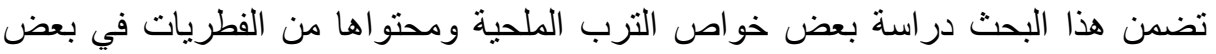

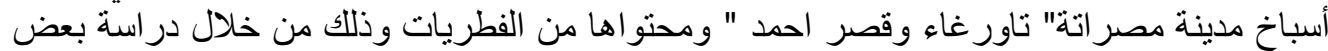

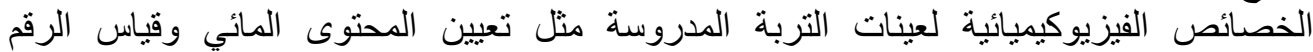
الهيدروجيني وتعيين كمية المواد العضوية وقياس كمية بعض العناصر وتعبين نوع التربة، كذللك تم عزل فطريات التربة بواسطة وسطي السابورود والتشابك وتم إجراء نفس الاختبار ات على عيلى عينات

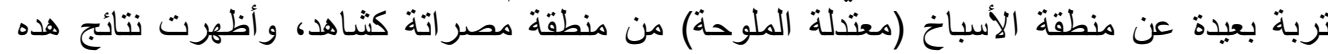

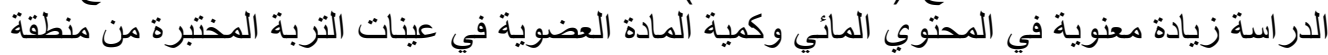

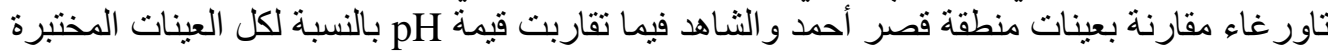

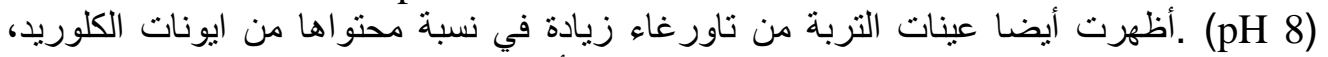

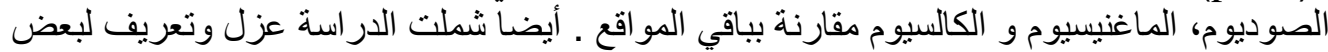
Limoniastrum monpetalum, الفطريات المتحملة للملوحة القريبة من المجموع الجذري لنباتات Juncus acutus وArtbrocnemum fruticosum بمدينة مصر اتة بليبيا . وقد تم تعريف 5 أنواع تنتمي إلى 5 أجناس، وكان فطر Aspergillus و

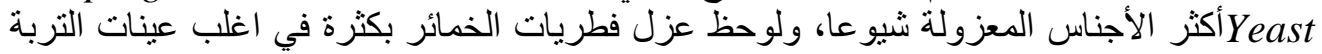

المالحة

مفاتيح الكلمات : سبخة، قصر احمد ، تاورغاء Yeast, Aspergillus

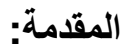

تقع منطقة تاور غاء في ظروف مناخية شبه جافة وتتأثر بدرجة مباثرة بمياه عين تاور غاء

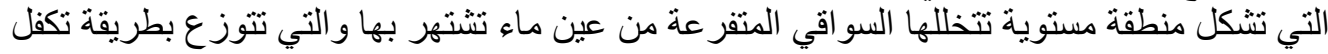
وصول مياه العين إلى كل منطقة وتنتهي في ثلاث مصبات رئيسية وتنتشر النباتات الملحية

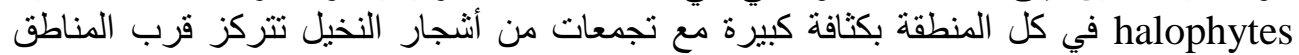

وتبلغ نسبة التغطية أكثر من 90\% ولا توجد سوى مساحات صغيرة خالية من النباتات كما توجد بعض السبخات الصغيرة التي تتجمع فيها المياه بين السواقي الرئيسية, ويتميز الغطاء النبات النباتي في

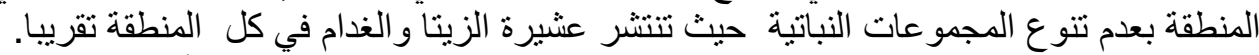

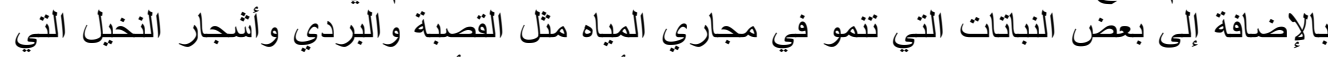
تثو اجد في مجموعات متناثرة ولا يوجد في المنطقة أي حوليات أو محاصيل حقلية سوى مساحات

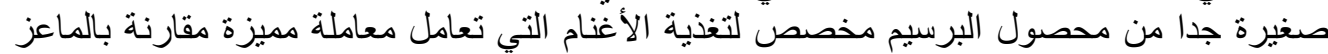

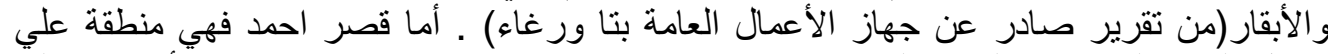

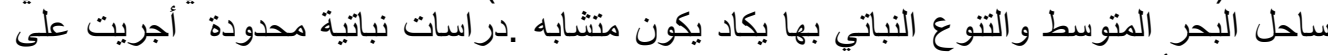

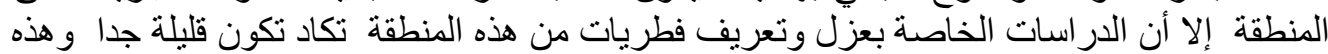

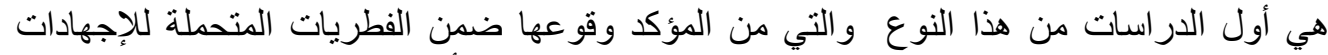
البيئية القاسية مثل الملوحة والقلوية والحرارة العالية مما يجعل لها أهمية اقتصادية عالية. ملح ملح كلوريد

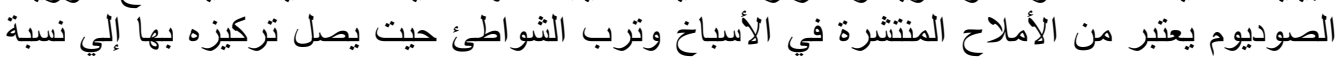
. (litchifield and Gillevet, 2002) \%ء. كما هو معروف أن اغلب الكائنات الدقيقة بما فيها الفطريات تستوطن البيئات المعتدلة الملوحة

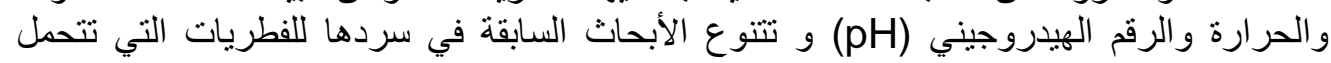
Fayoum J. Agric. Res. \& Dev., Vol. 27, No.1, January, 2013 
درجات عالية من الملوحة وبعض البيئات المالحة التي لا تتمو ا بها فطريات العديد من الأبحاث

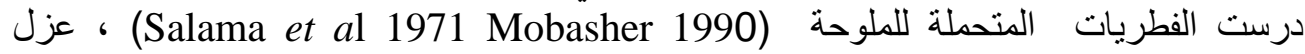

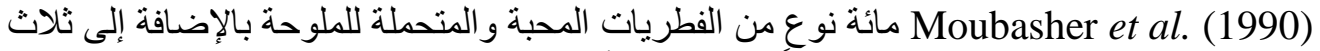

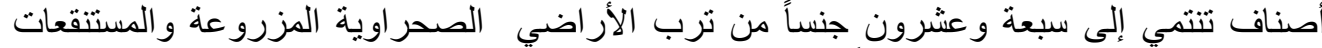

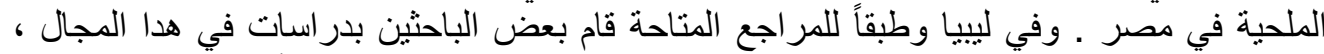
1967 Naim)

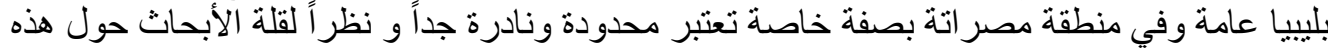

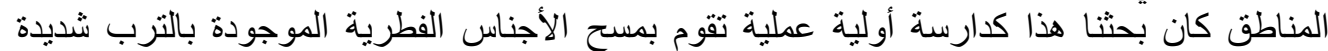
الملوحة في منطقة مصر اتخة بليبيا.

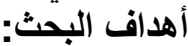

يهرف هذا البحث إلي دراسة بعض خواص تربة البه السبخة الملحية ومحتو اها من الفطريات في مدينة

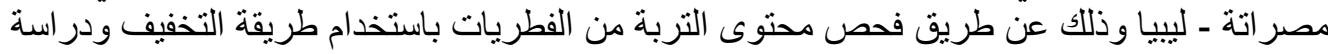

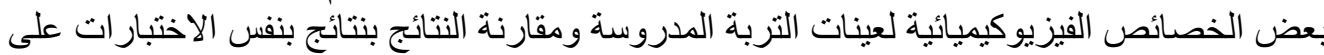
عينات تربة معندلة الملوحة كثاهد للمقارنة.

$$
\text { وصف المنطقة المدروسة }
$$

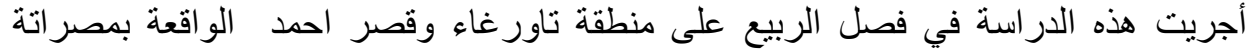

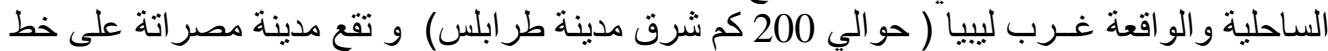
عرض 32,22 شمالا وخط طول 15,06 شرقا ، تهب الرياح في فصل الصني الصيف في هذه المنطقة من

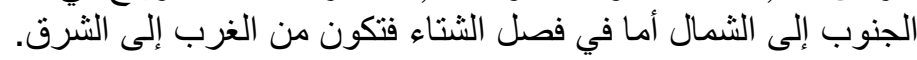

Limoniastrum تم اخذ عينات من التربة من المنطقة القريبة من المجموع الجذري لنبات النات طريقة جمع العينات النامية في سبخة منطقتي

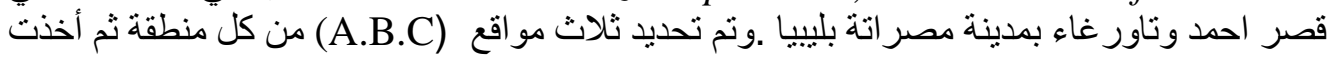
3 عينات ( من منطقة معندلة الملوحة من نفس مدينة مصر اتة كثاهد ) للمقارنة.

دراسة الخصائص الفيزيوكيميائية لعينات التربة تقدير المحتوي المائي

تم تعيين المحتوي المائي تبعا للطريقة المذكورة في المرجع (Chaturvedi and

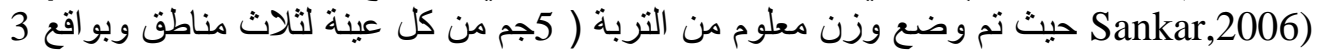

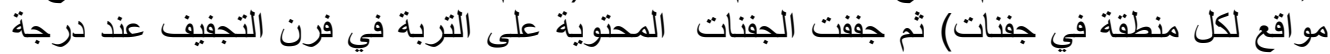

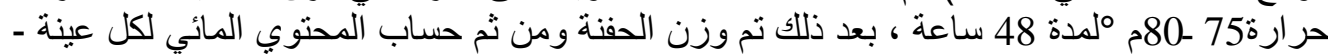
وقورنت النتائج بعينات الثناهد. قياس الرقم الهيروجيني (

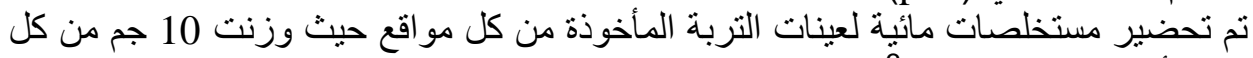

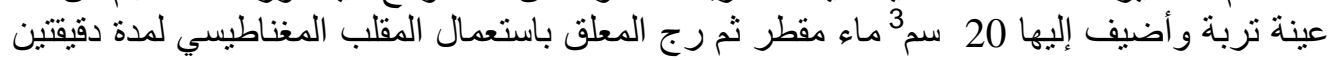

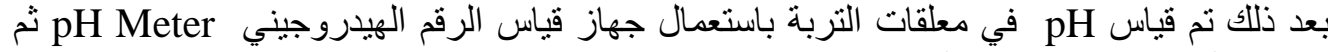
قورنت النتائج بنتائج عينات الثاهد (Chaturvedi, and Sankar, 2006). تقدير محتوى التربة من المواد العضو العضوية

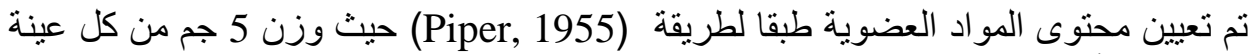

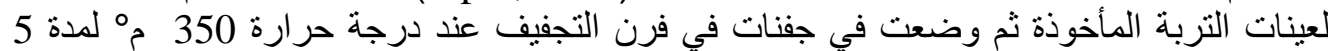

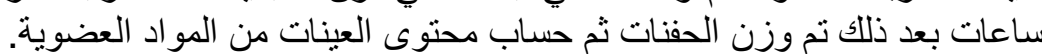

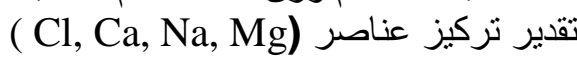

Fayoum J. Agric. Res. \& Dev., Vol. 27, No.1, January, 2013 
قدرت بعض العناصر (الصوديوم ، الكالسيوم ، الماغنيسيوم ، و الكلور) في مستخلص التربة

و عينه مرجعيـه (Blank) بعد هضـم العينـات باستخدام طريقة الترميد الرطب (Wet digestion) وفيها يستخدم حمض النيتريك المركز مع فوف أكسيد الهيدروجين وفق الطريقة المتبعة لذي, AOAC)

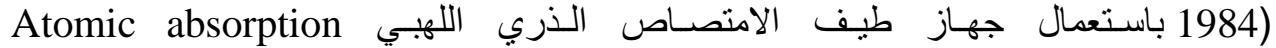

HITACHI .180-30, Equip No A-10 نوع Spectrophotometer Flame عزل وتعريف الفطريات في العينات المختبرة

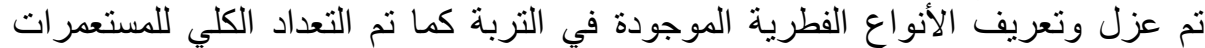
الفطرية النامية باستخدام طريقة أطباق التخفيف ( dilution method ) وبيئات اللوسط المغذي

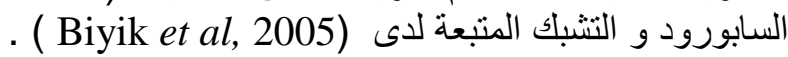
التحليل الإحصائي تم استخدام طريقة ANOVA one way لتحليل القراءي آتصات. النتائســج:

يبين الجدول رقم (1) النسبة المئوية للمحتوى المائي ومحتوى المواد العضوية وقيمة الأس

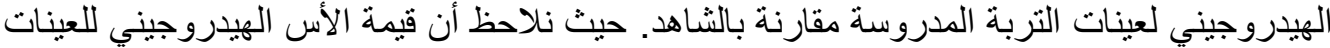

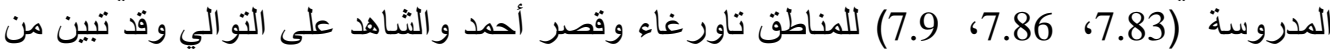

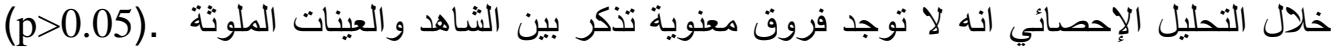

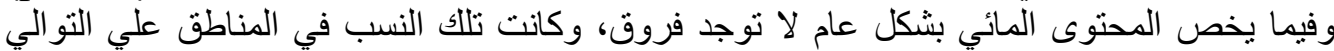

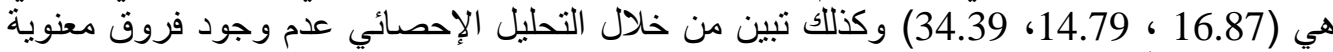

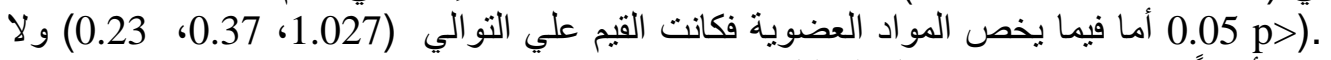

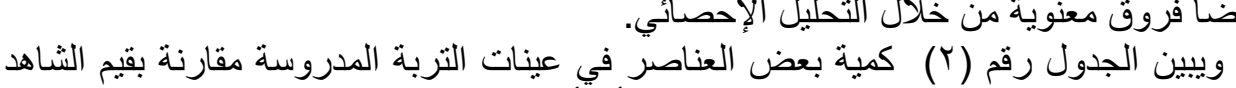
و هذه العناصر هي (Cl - Ca - Mg - Na) ونلاحظ أن أعلى كمية للعناصر هي الموجودة في منطقة

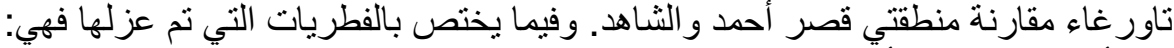

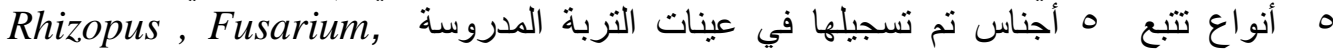
Aspergillus Penecillium , Yeast, من خلال الجدول رقم (3) والذي سجل فيه المسح المبدئي لأجناس وأنواع الفئس الفطريات المعزولة بواسطة الوسط المغذي السابورود لوحظ أن الجنس السائد في العينات هو

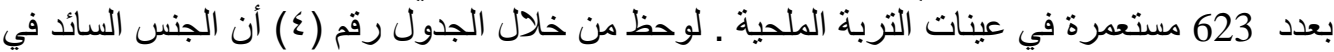

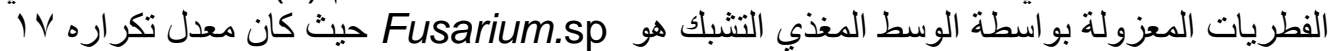

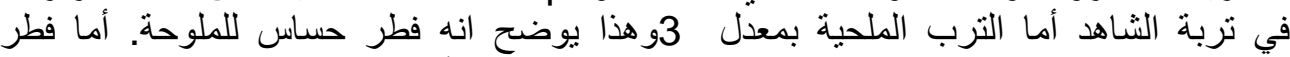
Aspergillus .niger.

جدول رقم ( () متوسط المحتوى المائي والأس الهيلروجيني وكمية المواد

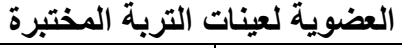

\begin{tabular}{|c|c|c|c|}
\hline الأس الهيدروجيني & كمية المواد العضوية & المحتوى المائي & المناطق المدروسة \\
\hline 7.83 & 2.824 & 45.74 & تاور غاء \\
\hline 7.86 & 0.802 & 29.88 & قصر أحمد \\
\hline 7.90 & 0.644 & 26.09 & الثاهد \\
\hline
\end{tabular}

جدول (Y) متوسط كمية العناصر لعينات التربة المختبرة (ملليجرام/كيلو جرام تربة)

Fayoum J. Agric. Res. \& Dev., Vol. 27, No.1, January, 2013 


\begin{tabular}{|c|c|c|c|c|}
\hline الصوديوم Na & الماغنيسيوم Mg & الكلوريد Cl & الكالسيوم Ca & المناطق \\
\hline 1624.9 & 532.89 & 9940.0 & 205.0 & تاور غاء \\
\hline 1031.2 & 187.5 & 1952.5 & 165.0 & قصر أحمد \\
\hline 354.2 & 11.32 & 443.75 & 72.5 & الثشاهد \\
\hline
\end{tabular}

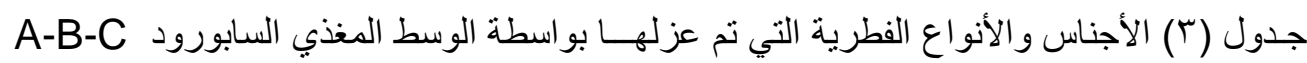

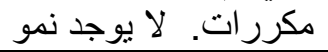

\begin{tabular}{|c|c|c|c|c|c|c|c|c|c|}
\hline \multirow{2}{*}{ اسم الفطر } & \multicolumn{3}{|c|}{ ت تاور غاء } & \multicolumn{3}{|c|}{ قصر أحمد } & \multicolumn{3}{|c|}{ الشاهد } \\
\hline & A1 & A2 & A3 & B1 & B2 & B3 & C1 & $\mathrm{C2}$ & $\mathrm{C3}$ \\
\hline Fusarium & 5 & 2 & - & 2 & 1 & 1 & 6 & 6 & 5 \\
\hline Aspergillus .niger & 3 & - & - & 12 & 4 & - & 1 & 2 & 2 \\
\hline Yeast & 450 & 120 & 400 & 300 & - & - & 2 & 2 & - \\
\hline Penecillium.sp & - & - & - & 2 & 1 & 5 & - & - & - \\
\hline إجمالي عدد المستعمرات & 458 & 122 & 400 & 316 & 6 & 6 & 9 & 10 & 7 \\
\hline إجمالي عدد الأنواع & 3 & 2 & 1 & 4 & 3 & 2 & 3 & 3 & 2 \\
\hline إجمالي عدد ألأجناس & 3 & 2 & 1 & 4 & 3 & 2 & 3 & 3 & 2 \\
\hline
\end{tabular}

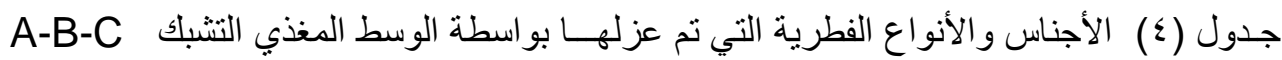

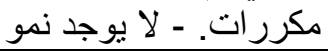

\begin{tabular}{|c|c|c|c|c|c|c|c|c|c|}
\hline \multirow[t]{2}{*}{ اسم الفطــــر } & \multicolumn{3}{|c|}{ تاور غاء } & \multicolumn{3}{|c|}{ قصر أحمد } & \multicolumn{3}{|c|}{ الثـاهد } \\
\hline & A1 & A2 & A3 & B1 & B2 & B3 & $\mathrm{C} 1$ & $\mathrm{C} 2$ & $\mathrm{C} 3$ \\
\hline Aspergillus .niger. & 16 & 1 & 10 & 4 & 12 & 11 & 6 & 6 & 5 \\
\hline Rhizopus. sp & 1 & 2 & - & - & - & - & 1 & 2 & 2 \\
\hline Penecillium. sp & 2 & - & 10 & 5 & 5 & 3 & 2 & 2 & - \\
\hline Fusarium .sp & 3 & 1 & - & 1 & - & 5 & - & - & - \\
\hline إجمالي عدد المستعمر ات & 22 & 4 & 20 & 10 & 17 & 19 & 9 & 10 & 7 \\
\hline إِّمالي عدد الأنواع & 4 & 4 & 2 & 3 & 2 & 3 & 3 & 3 & 2 \\
\hline إجمالي عدد الأجناس & 4 & 3 & 2 & 3 & 2 & 3 & 3 & 3 & 2 \\
\hline
\end{tabular}

\section{الاراسات الفيزيوكيميائية علي عينات التربة:}

أظهرت النتائج أن الأس الهيدروجيني للعينات المختبرة هو قلوي خفيف الهيف بمتوسط 7.86 حيث

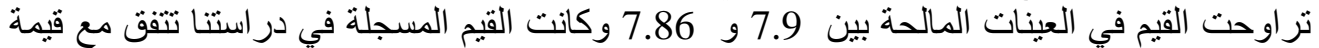

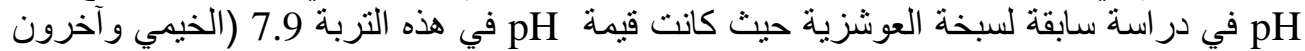

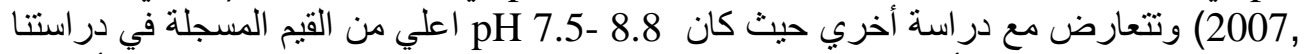

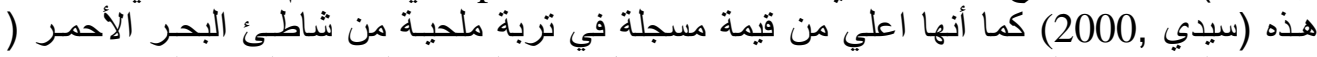

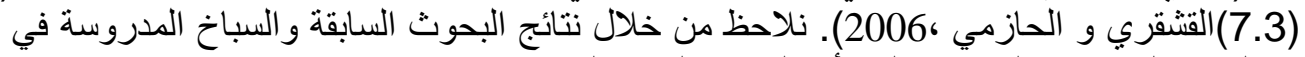

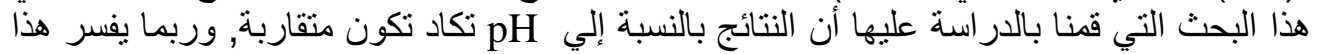

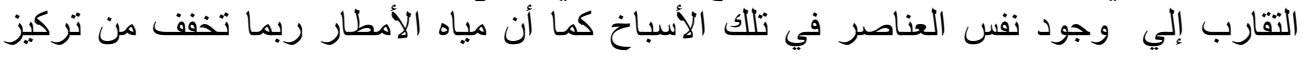

Fayoum J. Agric. Res. \& Dev., Vol. 27, No.1, January, 2013 


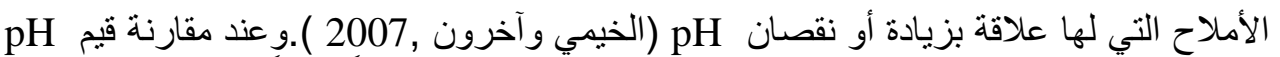

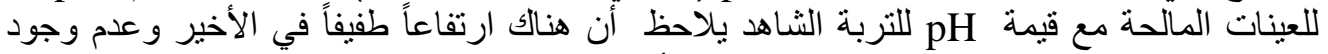

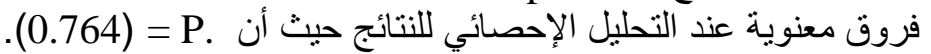

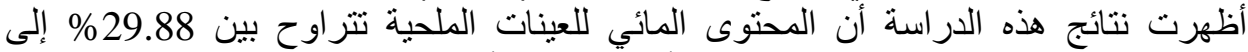

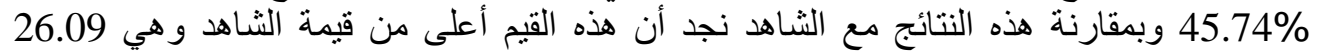

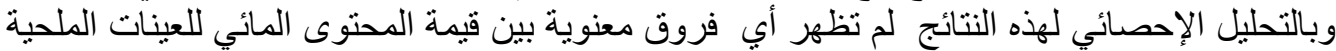

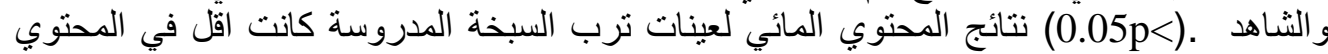

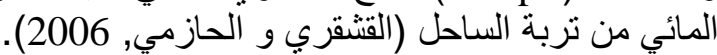

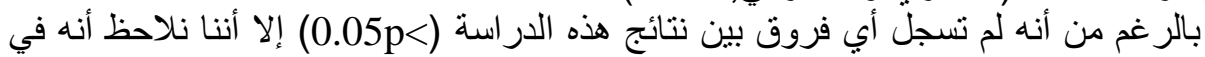

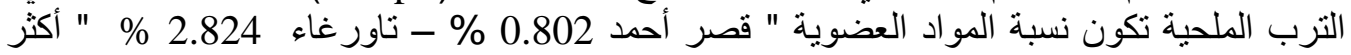

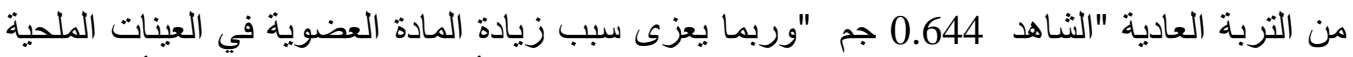

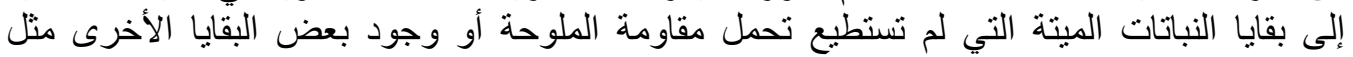

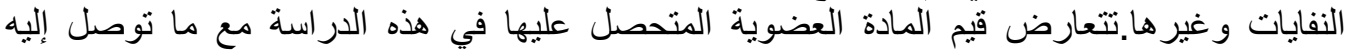

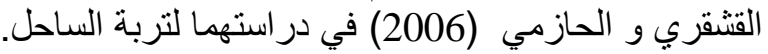

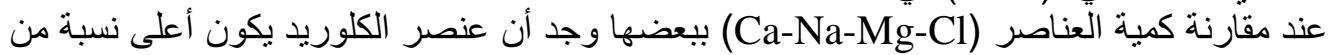

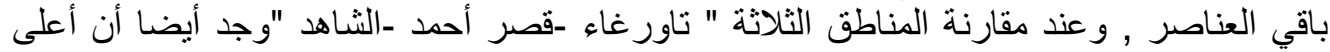

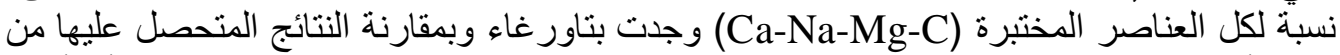

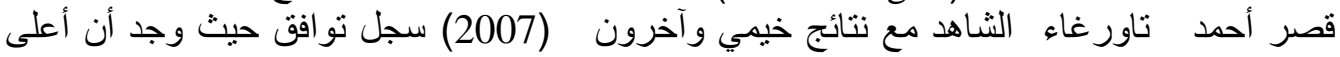

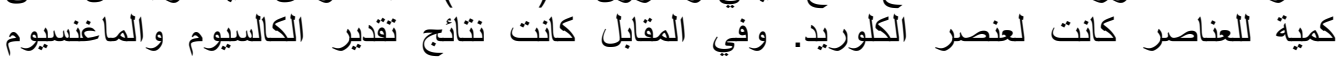

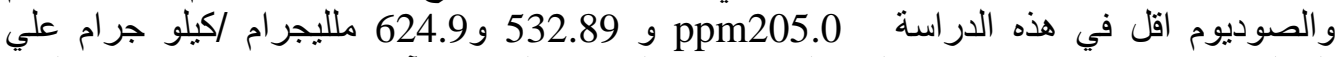

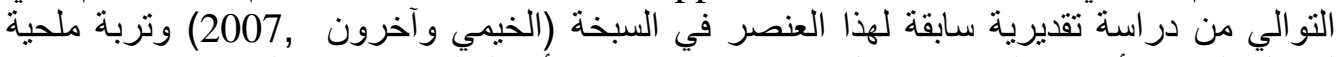

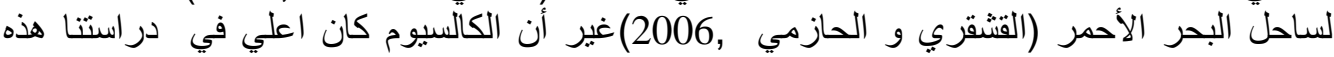

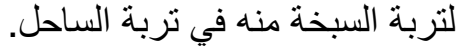
الفطريات المززولة من التربة:

سجل عند عزل الفطريات بواسطة الوسط المغذي السابورود وجود 4 أنواع هي ني Aspergillus.niger Yeast.sp - Penecillium sp - Fusarium.sp

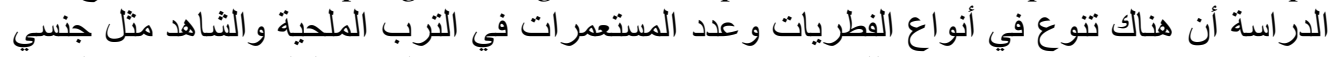
Aspergillus.niger-Yeast_

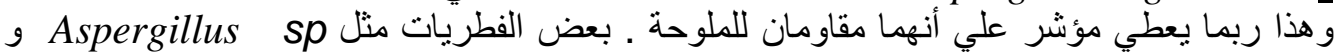

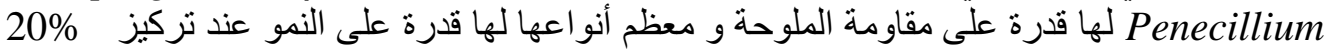

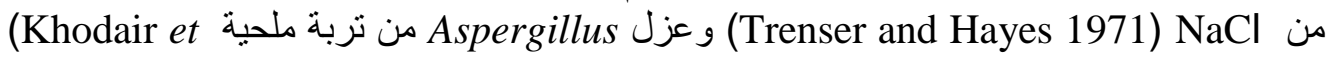
.al., 1991)

مع أن Aspergillus.niger عزل من ترب قلوية pH في مناطق عدة من العالم من ضمنها

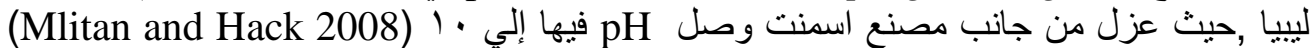

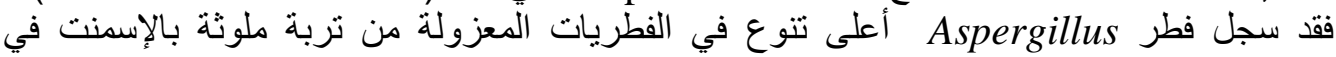

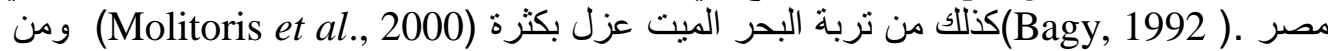
الصحر اء الكبرى بالسعودية (Abdel Hafez, 1982) إضافة إلي أن هذا الفطر عزل من من تربة

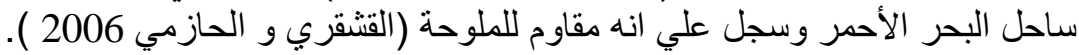

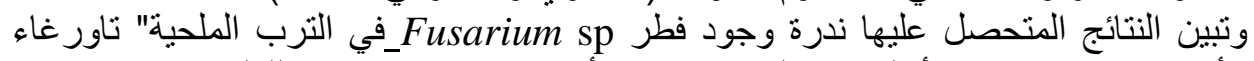

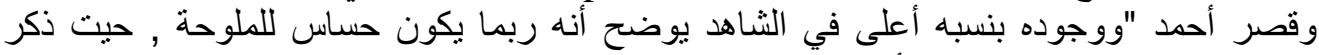

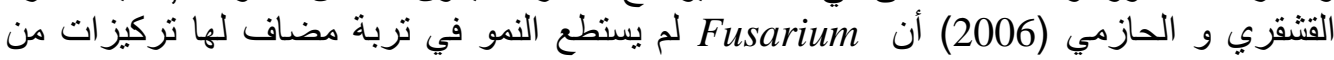

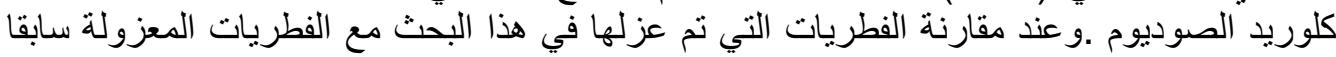

Fayoum J. Agric. Res. \& Dev., Vol. 27, No.1, January, 2013 
في ترب سبخة (خيمي وآخرون، 2007) نلاحظ وجود اتفاق في 3 أنواع من الفطريات وهي Aspergillus.niger - Penecillium $\mathrm{sp}-$ Yeast $\mathrm{sp}$

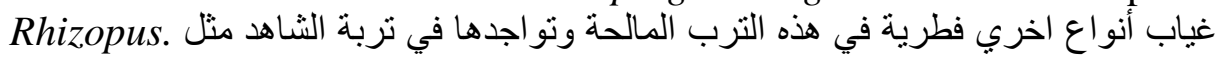

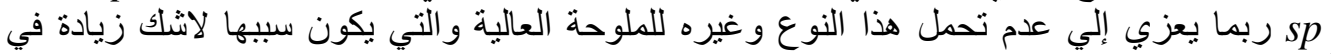
أملاح الصوديوم ,ألبوتاسيوم ,الماغنسيوم , الحديد و البورون . (Steiman et al., 1995) حيث

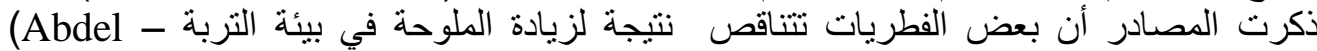

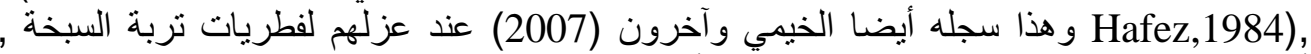
و أيضا (Steiman et al., 1995). كما سجل أن مجتمعات التربة الملحية تفتقر إلي التنوع الفطري .(Moustafa and Al- Musallam 1975)

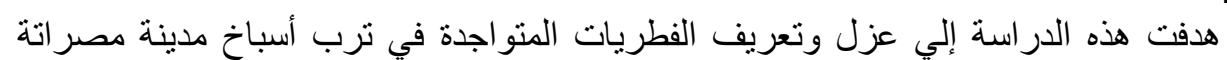

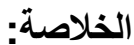

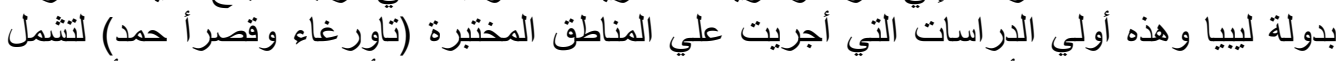

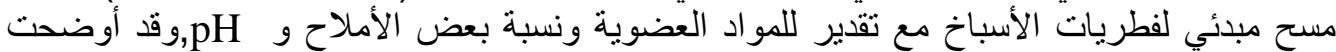

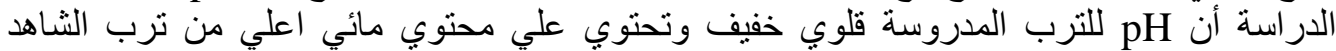

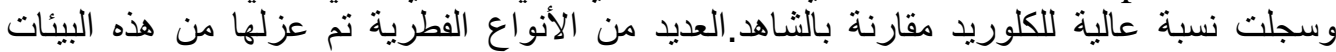
المالحة بالرغم من أن العديد من الفطريات لا تفضل النمو في الظروف الفراع القلوية السائدة في السبخة.

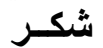

أتقدم بالثكر إلي جامعة مصر اتة علي دعمها لهذه الدراسـة من خلال تحليل عينات التربـة وتعريف الفطريات في معاملها.

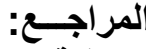

ـ رقية محمد القثقري ونوال عيسي الحازمي (2006) تأثير الملوحة علي تو اجد الفطريات في تربـة ساحل البحر الاحمر لمديينة القنفذة بالمملكة العربية السعودية.مجلة اسيوط لعلية لعلوم البئية البئة مجلد 9,1

ـ صلوح محمد سيدي (2000) در اسة الغطاء النباتي في سبخة قصر أحمد بمصر اته. شعبة النبات، كلية الاداب و العلوم مصر اته. (2007) درائة

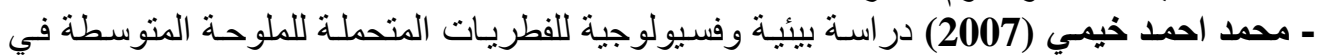

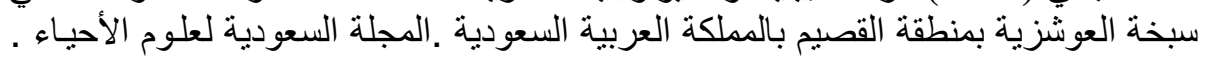

14(2) 115-123.

Abdel-Hafez, S.I. (1982): Osmophilic fungi of desert soils in Saudi Arabia. Mycopath.,80: pp9-14.

Abdel-Hafez, S.I. (1984): Survey of airborne fungus spores at Taif, Saudi Arabia. Mycopath., 88: pp39-44.

Bagy, M.M, (1992): Saprophytic and keratinophilic fungi isolated from desert and cultivated soils continuously exposed to cement dust particles in Egypt. Zentralblatt für Mikrobiologie 147:pp 418-426.

Biyik, H. Imali A, Atalan E, Tufenkci S, Ogun E, (2005): Diversity of microfungi in soil polluted by cement factory. Fresenius Environmental Bulletin 14 :pp 130-137.

Chaturvedi, R.K, Sankar K, (2006): Laboratory manual for the physicochemical analysis of soil, water and plant. Wildlife Institute of India, Dehradun.

Fayoum J. Agric. Res. \& Dev., Vol. 27, No.1, January, 2013 
Khodair, A.A.; Ramadani, A.S. and Aggab, A.M. (1991): Occurrence and density of alkalophilic bacteria and fungi in saline soils of Makhah district, Arab Gulf J. of Sci. Res., 9 (3): pp 119-132.

Litchfield, C.D. and Gillevet, P.M. (2002): Microbiol diversity and complexity in hypersaline environments. A. preliminary assessment. J. Indust. MicroBio. and Biotech. 28: pp48-55.

Mlitan, A. and Hack E. (2008): Effects of some cement constituents on growth of Aspergillus nidulans. The 163 rd SGM Trinity college Dublin meeting, 8-11 September.

Moubasher, A.H., Abdel-Hafez, S.I., Bagy, M.M. and Abdel-Sater, M.A. (1990): Halophilic and halotlerant fungi in cultivated, desert, and salt marsh soils from Egypt. Acta Mycol., xxvi (2): pp65-81.

Moustafa, A.F. and Al-Musallam, A.A. (1975): Contribution to the fungul flora of Kuwait. Trans. Br. Mycol. Soc., 65 (3): pp547-553.

Naim M.S, (1967): Contribution to the knowledge of soil fungi in Libya - II. Fungus flora under citrus trees in Libya. Mycopathologia et Mycologia Applicata 31, pp300-304.

Piper, C.S. (1955): Soil and plant analysis. A laboratory manual of methods for the examination of soil and determination of the inorganic substituents of plants. Inter. Pub. Inc., New York.

Salama, A.L. ; EL-Batanoni, K. and Ali, M.I. (1971): Studies Mediterranean Coast and Lybian Desert U. A. R., J. Bot., 14:pp99-114.

Steiman R., Guiraud P., Sage L. and Seigle-Murandi F. (1995): Mycoflora of soil around the Dead Sea. I. Ascomycetes (including Aspergillus and pincillium), Basidiomycetes, Zygomycetes. Syst. Appl. Microbiol. 18: pp310-317.

Trenser, H.D. and Hayes, J.A. (1971): Sodium chloride tolerance of terrestrial fungi. Appl. Microbiol., 22: pp210-213.

\title{
STUDY OF SOME PHYSIOCHEMICAL CHARACTERISTICS AND FUNGAL IDENTIFICATION IN SALTS SOIL COLLECTED FROM TAWRGA AND KASHER AHMED REGIONS IN LIBYA.
}

\author{
A.B. Mlitan ${ }^{1}$, E.H. Elbakosh ${ }^{2}$, S.A. Safar ${ }^{3}$ and A.M. Abo- Alayem ${ }^{4}$ \\ ${ }^{1-4 *}$ Department of Botany, Faculty of Science, University of Misurata \\ aefmsr20102@yahoo.com ${ }^{1}$
}

\begin{abstract}
The aim of this work was to study some physiochemical characteristics in salt affected soils collected from Tawrga and Kasher Ahmed regions. The study includes determination of $\mathrm{pH}$, water content, organic matter and some elements content also fungal identification. The obtained results showed that all soil samples were slightly alkaline in reaction and containing low concentration of organic matter $0.802 \mathrm{~g}-2.824 \mathrm{~g}$. Soil water content ranged
\end{abstract}

Fayoum J. Agric. Res. \& Dev., Vol. 27, No.1, January, 2013 
$\wedge$

from $\% 29.88$ to $45.74 \%$. Soil $\mathrm{pH}$ ranged between 7.86 to 7.9 with no appreciable differences between localities. Soil samples differed in their content of elements. Most tested localities contained high amounts of $\mathrm{Cl}$ compared to normal soil samples. All tested elements recorded high contents in samples collected from Tawrga. Five genuses belong to five species including yeasts were identified. The most frequent genus was Yeasts from which high number of colonies isolates were detected. In addition Aspergillus were isolated. Aspergillus and Yeasts may be considered one of the resistant fungi to high soil salinity $(\mathrm{NaCl})$ due to its large colonies numbers isolated from these areas.

Key words: Yeast, Na, Libya.

Fayoum J. Agric. Res. \& Dev., Vol. 27, No.1, January, 2013 